\title{
Theoretical Electronic Structure of the Lowest-Lying Electronic States of the $\mathrm{CaCl}$ Molecule
}

\author{
H. Jawhari ${ }^{1}$, M. Korek ${ }^{1}$, R. Awad ${ }^{2} \&$ M. R. Sakr ${ }^{2}$ \\ ${ }^{1}$ Faculty of Science, Beirut Arab University, Riad El Solh, Beirut, Lebanon \\ ${ }^{2}$ Physics Department, Faculty of Science, Alexandria University, Egypt \\ Correspondence: M. Korek, Faculty of Science Beirut Arab University, P.O.Box 11-5020, Beirut, Lebanon. Tel: \\ 961-3-747617. E-mail: fkorek@yahoo.com
}

\author{
Received: June 4, 2014 Accepted: June 23, 2014 Online Published: July 9, 2014 \\ doi:10.5539/apr.v6n4p97 \\ URL: http://dx.doi.org/10.5539/apr.v6n4p97
}

\begin{abstract}
The potential energy curves of 10 doublet and 5 quartet low-lying electronic states of $\mathrm{CaCl}$ molecule have been investigated by using the Complete Active Space Self Consistent Field (CASSCF) with Multi Reference Configuration Interaction (MRCI) using effective core potential for both atoms. Based on the investigated PECs, the harmonic frequency $\omega_{e}$, the internuclear distance $R_{e}$, the dipole moment, and the electronic energy with respect to the ground state $T_{e}$ have been calculated for the considered electronic states. A rovibrational study has been performed with the calculation of the eigenvalue $E_{v}$, the rotational constant $B_{v}$ and the abscissas of the turning points $R_{\min }$ and $R_{\max }$. The comparison between the values of the present work and those available in the literature for several electronic states shows a very good agreement. Nine new excited electronic states have been investigated here for the first time.
\end{abstract}

Keywords: $a b$ initio calculation, $\mathrm{CaCl}$ molecule, potential energy curves, spectroscopic constants, dipole moment, rovibrational calculation

\section{Introduction}

Alkaline earth monohalides have received, in recent years, considerable attention in addition to their importance in high-temperature chemistry (D'incan et al., 1991; Verges et al., 1993; Kaledin et al., 1999; Childs et al., 1984) and chemiluminescent reactions (Amiot et al 1991; Langhoff et al., 1986; Menzinger et al 1974; Yang et al., 2004; Handy et al., 1989; Dykstra, 1977). Accurate experimental and theoretical data have become available for these monohalides (Ernst et al 1984; Harris et al., 1993; Murphy et al., 1990, 1995; Berg et al., 1993; Bündgen et al 1991). The spectroscopic constants of the $\mathrm{B}^{2} \Sigma^{+}-\mathrm{X}^{2} \Sigma^{+}$systems are obtained for the first time by using high resolution laser excitation spectroscopy by (Domaille et al., 1977). A rotational analysis of the $\mathrm{E}^{2} \Sigma^{+}-\mathrm{X}^{2} \Sigma^{+}$ transition of $\mathrm{CaCl}$ is made by (Berg et al., 1978). Experimental potential energy curves have been constructed for the first time for $(\mathrm{X})^{2} \Sigma^{+}$and $(\mathrm{C})^{2} \Pi$ electronic states of $\mathrm{CaCl}$ molecule by (Rao et al., 1983). Energies and electric dipole moments of the low lying electronic states of the alkaline earth monohalides, by applying an electrostatic polarization model, are investigated by (Torring et al., 1989). Transition energies and permanent and transition dipole moments, of the low-lying states of calcium monohalides, have been evaluated by (Allouche et al., 1993) using the ligand-field approach, proposed by (Rice et al., 1985).

We investigate in the present work the Potential Energy Curves (PECs) for the 15 low-lying electronic states with the high level MRCI calculation. The spectroscopic constants $T_{e}, R_{e}, \omega_{e}$ and $B_{e}$ of the molecule CaCl have been obtained for the investigated doublet electronic states along with the static dipole moment, the eigenvalue $E_{v}$, the rotational constant $B_{v}$, and the abscissas of the turning points $R_{\min }$ and $R_{\max }$.

\section{Method}

An $a b$ initio calculations were performed for $\mathrm{CaCl}$ molecule using the multireference configuration interaction method (MRCI) for the ground and low-lying doublet and quartet electronic states. These calculations were preceded by Multi-configuration self-consistent field (MCSCF) calculations (Werner \& Knowles, 1985; Knowles $\&$ Werner, 1985) in which all valence molecular orbitals were optimized. All calculations were performed with the MOLPRO program package (Molpro) taking advantage of the graphical user interface GABEDIT (Allouch, 2011). Calcium and Chlorine atoms are treated through pseudo potentials approach. Effective Core 
Potential-MWB basis set are chosen for both atoms from library of the MOLPRO software. Through the MCSCF/MRCI calculations, the wave function is under $C_{2 v}$ symmetry. The $1 s^{2} 2 s^{2} 2 p^{6}$ of both atoms were frozen in the MCSCF procedure. The number of closed-shell orbitals is $7\left(3 \sigma, 2 \pi_{x}, 2 \pi_{y}, 0 \delta\right)$ and the number of active orbitals is 5 . The energies of PECs are calculated in the range of internuclear distances $1.5 \leq \mathrm{R} \leq 11.5 \AA$.

\section{Results}

The calculations for the doublet and quartet PECs, for the lowest 15 electronic states of the CaCl molecule, have been performed for the internuclear range $1.5 \AA \leq \mathrm{R} \leq 11.5 \AA$ in the representation ${ }^{2 \mathrm{~s}^{+1}} \Lambda^{(+/)}$(Figures 1-3).

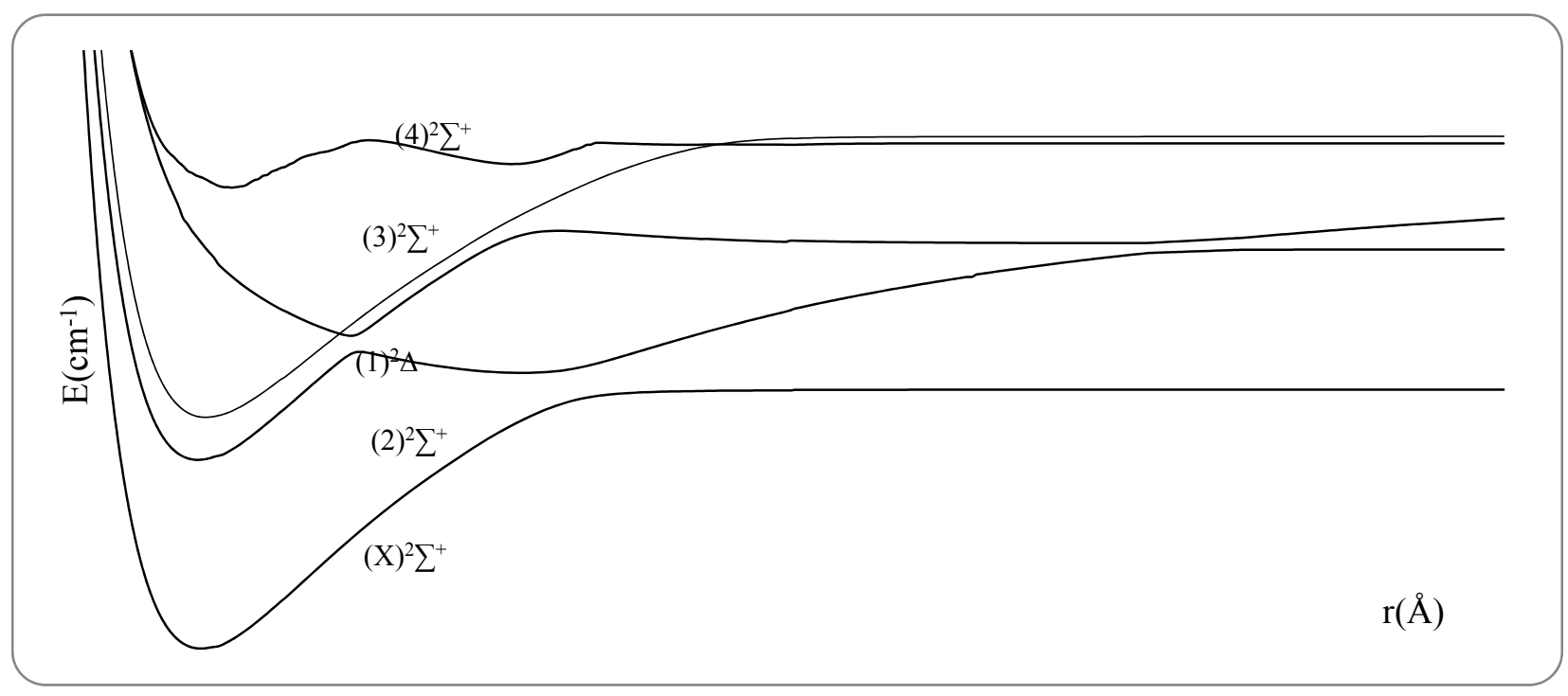

Figure 1. Potential energy curves for the ${ }^{2} \Sigma^{+},{ }^{2} \Delta$ and ${ }^{2} \Sigma^{-}$states of the molecule CaCl

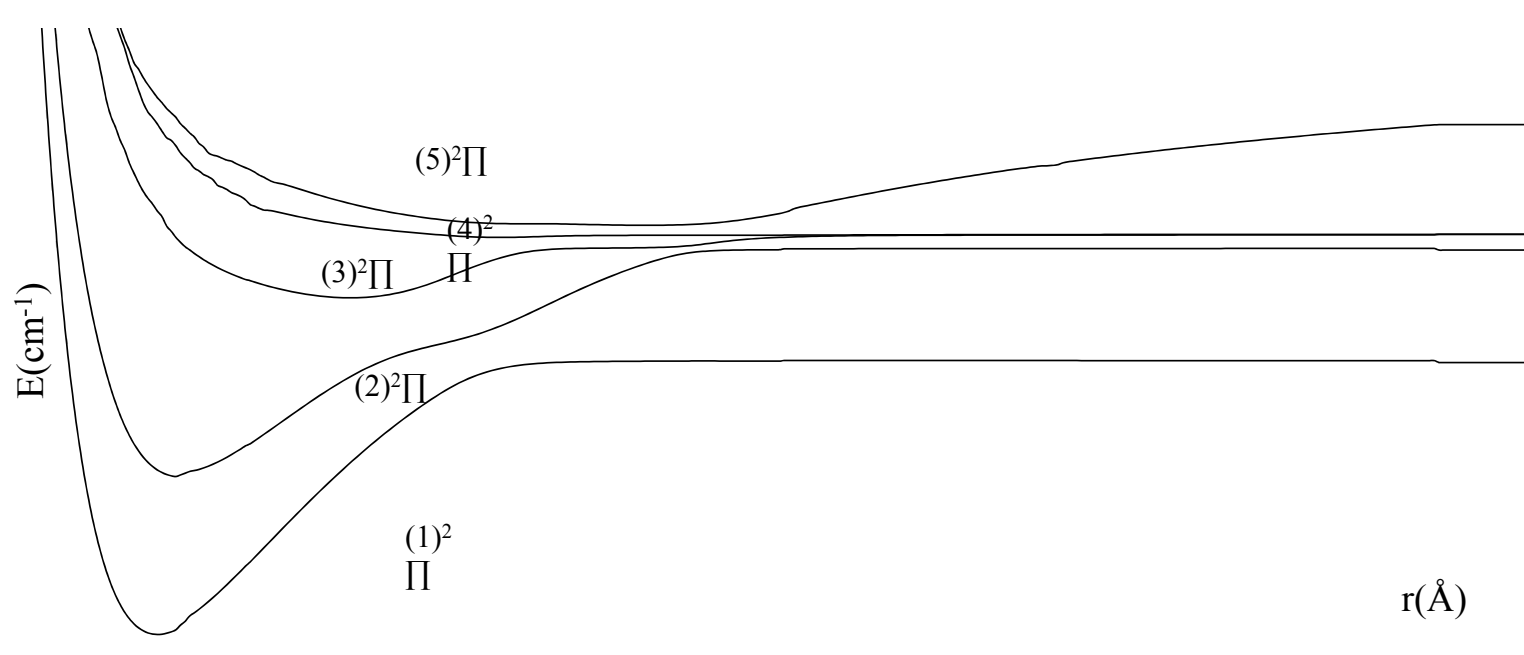

Figure 2. Potential energy curves for the ${ }^{2} \Pi$ states of the molecule $\mathrm{CaCl}$ 


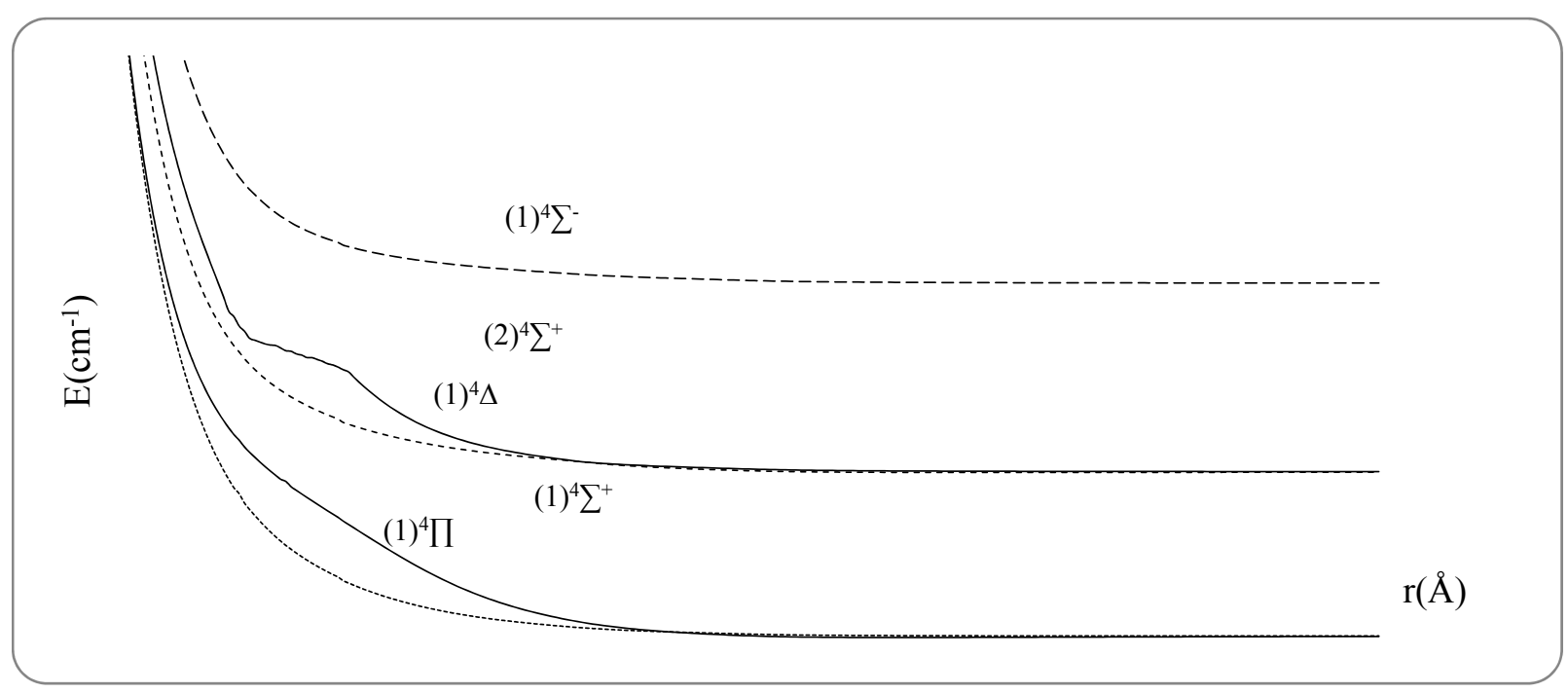

Figure 3. Potential energy curves for the ${ }^{4} \Sigma^{+},{ }^{4} \Delta,{ }^{4} \Pi$ and ${ }^{4} \Sigma^{-}$states of the molecule $\mathrm{CaCl}$

The PECs of the low-lying electronic states intercross each other in several places which are displayed in Table 1 for all the considered electronic states.

Table 1. Positions of the crossing and avoided crossings between the different electronic states of the molecule $\mathrm{CaCl}$

\begin{tabular}{lcccc}
\hline \multirow{2}{*}{ State1 } & \multirow{2}{*}{ State2 } & crossing & \multicolumn{2}{c}{ Avoided crossing } \\
\cline { 3 - 5 } & & $\mathrm{r}_{\mathrm{c}}(\AA)$ & $\mathrm{r}_{\mathrm{av}}(\AA)$ & $\Delta \mathrm{E}_{\mathrm{av}}\left(\mathrm{cm}^{-1}\right)$ \\
\hline$(1)^{2} \Sigma^{+}$ & $(2)^{2} \Sigma^{+}$ & & 5.11 & 3161.8 \\
& & & 3.58 & 1718.7 \\
$(2)^{2} \Sigma^{+}$ & $(3)^{2} \Sigma^{+}$ & & and & and \\
& & & 8.95 & 1006.8 \\
$(1)^{2} \Delta$ & $(3)^{2} \Sigma^{+}$ & 3.47 & & \\
$(1)^{2} \Delta$ & $(4)^{2} \sum^{+}$ & 6.03 & & \\
$(1)^{2} \Pi$ & $(2)^{2} \Pi$ & & 4.60 & 3588.3 \\
$(2)^{2} \Pi$ & $(3)^{2} \Pi$ & & 5.98 & 601.7 \\
$(1)^{4} \Sigma^{+}$ & $(1)^{4} \Pi$ & 5.07 & & \\
\hline
\end{tabular}

In order to calculate the spectroscopic constants: transition energy with respect to the energy minimum for the ground state $T_{e}$, equilibrium internuclear distance $R_{e}$, harmonic frequency $\omega_{e}$ and the rotational constants $B_{e}$, the calculated potential energy values were fitted to a polynomial in $\mathrm{R}$ around the minimum. These values along with the experimental and theoretical data available in literature are displayed in Table 2. Our MRCI computational equilibrium position is in good agreement compared with the experimental value of (Allouche et al., 1993) with a discrepant relative difference $\delta \mathrm{R}_{\mathrm{e}} / \mathrm{R}_{\mathrm{e}}=3.7 \%$ for $(\mathrm{X})^{2} \Sigma^{+}$state. The comparison of the harmonic frequency $\omega_{\mathrm{e}}$, calculated in the present work for the ground state, is in acceptable agreement by comparing to those given by (Domaille et al., 1977; Berg et al., 1978) with the relative differences $8.6 \%$ and $8.9 \%$ respectively. Similar results are obtained by comparing our calculated value of $B_{e}$ to those given in literature (Domaille et al., 1977; Berg et al., 1978 ) where the relative differences are $7.8 \%$ for $(\mathrm{X})^{2} \Sigma^{+}$state and $7.7 \%$ for $(2)^{2} \Sigma^{+}$state. 
Table 2. Spectroscopic parameters $T_{e}\left(\mathrm{~cm}^{-1}\right), \mathrm{R}_{\mathrm{e}}(\AA), \omega_{\mathrm{e}}\left(\mathrm{cm}^{-1}\right)$ and $\mathrm{B}_{\mathrm{e}}\left(\mathrm{cm}^{-1}\right)$ for the ground and low-lying excited ${ }^{2} \Lambda^{(+)}$states of $\mathrm{CaCl}$ molecule

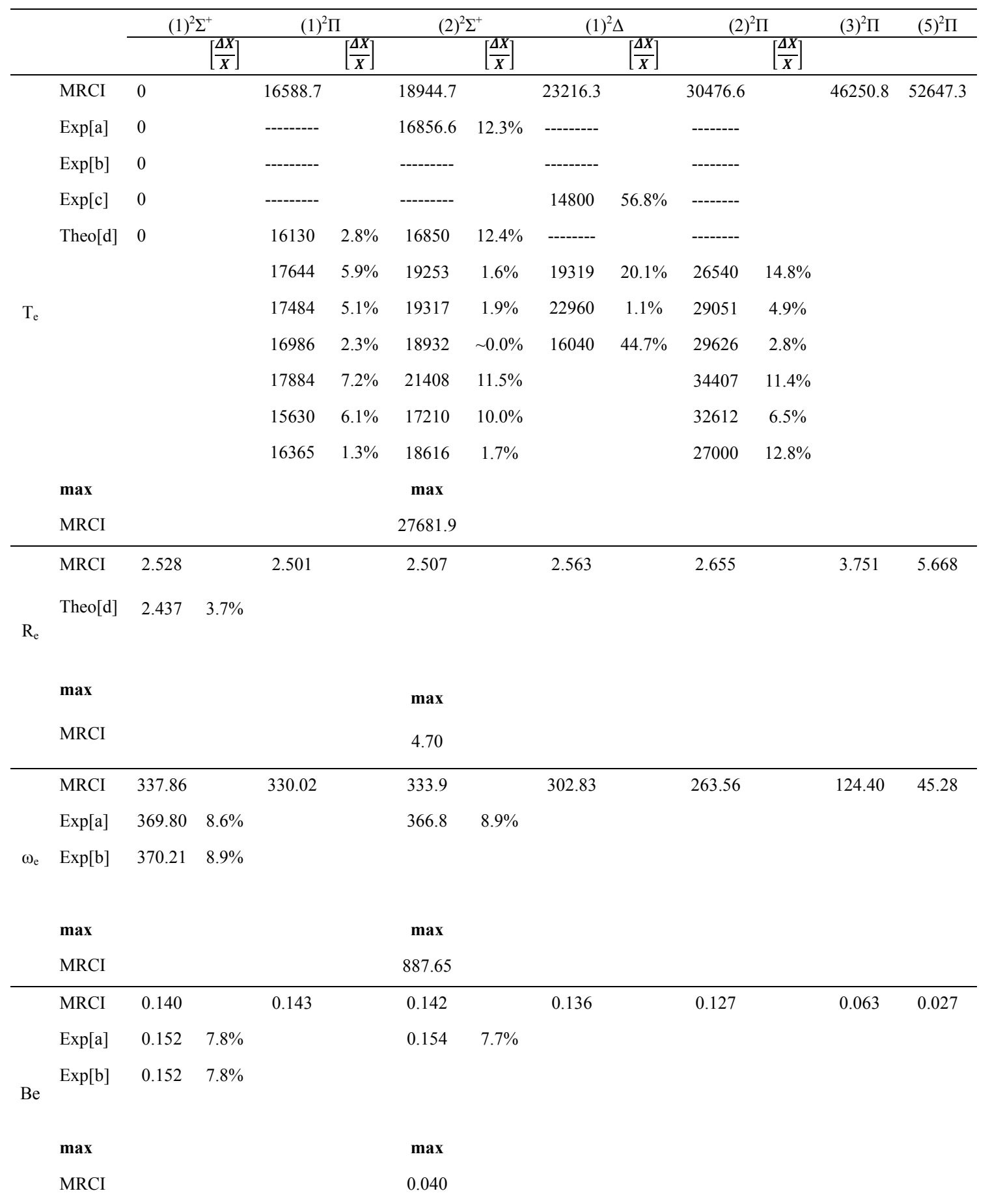

${ }^{(a)}$ Domaille et al., 1977, ${ }^{(b)}$ Berg et al., 1978, ${ }^{\left({ }^{(c)}\right.}$ Klynning et al., 1981, ${ }^{(\mathrm{d})}$ Allouche, et al., 1993.

The comparison of our calculated values of $T_{e}$ for the different investigated electronic states with the available experimental data in literature (Rice et al., 1985; Domaille et al., 1977; Huber et al., 1978) shows a very good agreement for the first excited states $(1)^{2} \Pi$ with relative difference $\delta \mathrm{T}_{\mathrm{e}} / \mathrm{T}_{\mathrm{e}}=2.8 \%$ and this agreement becomes larger for the 2 excited electronic states $(2)^{2} \Sigma^{+}$and $(2)^{2} \Pi$ with the relative differences $12.4 \%$ and $14.8 \%$ respectively. The discrepancy between our calculated values of $T_{e}$ with the theoretical calculated values of the literature (Allouche et al., 1993)depends on the technique of calculation of these values; while the relative difference is $0.0 \% \leq \delta \mathrm{T}_{\mathrm{e}} / \mathrm{T}_{\mathrm{e}} \leq 6.5 \%$ using some techniques (Rice et al., 1985; Torring et al., 1989, with or without 
polarization) for the electronic states $(1)^{2} \Pi,(2)^{2} \Sigma^{+}$and $(2)^{2} \Pi$ this relative difference becomes $7.2 \% \leq \delta \mathrm{T}_{\mathrm{e}} / \mathrm{T}_{\mathrm{e}} \leq 44.7 \%$ for other techniques (Rice et al., 1985; Torring et al., 1989, with or without polarization) of calculation (Allouche, et al., 1993). One can notice that, by using one technique of calculation (Rice et al., 1985; Torring et al., 1989) there is a very good agreement with our results for the state $(2)^{2} \Sigma^{+}$where the relative difference is $\delta \mathrm{T}_{\mathrm{e}} / \mathrm{T}_{\mathrm{e}}=0.0 \%$ while this agreement deteriorate by using the same technique of calculation for the state $(1)^{2} \Delta$ where relative difference is $\delta \mathrm{T}_{\mathrm{e}} / \mathrm{T}_{\mathrm{e}}=44.7 \%$. The largest relative difference for this state is $56.8 \%$ which is corresponding to the value obtained by (Klynning et al., 1981).

By using the canonical functions approach (Kobeissi et al., 1989; Korek et al., 1992; Korek et al., 1999), the eigenvalue $E_{v}$, the rotational constant $B_{v}$, the centrifugal distortion constant $D_{v}$, and the abscissas of the turning point $R_{\min }$ and $R_{\max }$ have been calculated for the investigated electronic states. Table 3 displays these results for the ground state up to $v=101$ as a sample (the data for the other states are available with authors). The comparison of our calculated values of $E_{v}, R_{\min }$ and $R_{\max }$ to those obtained by (Rao et al., 1983) for the two states $(X)^{2} \Sigma^{+}$and $(2)^{2} \Pi$, shows that our calculated values for $E_{v}$ are systematically larger with an average relative difference of $14.5 \%$ while our calculated values for $\mathrm{R}_{\min }$ and $\mathrm{R}_{\max }$ are in good agreement with an average relative difference $4.3 \%$.

Table 3. Values of the eigenvalue $E_{v}$, the rotational constant $B_{v}$, the centrifugal distortion constants $D_{v}\left(\right.$ in $\mathrm{cm}^{-1}$ ) and the abscissas of the turning points (in $\AA$ ) of the $(\mathrm{X})^{2} \Sigma^{+}$state of $\mathrm{CaCl}$ molecule up to $\mathrm{v}=10$

\begin{tabular}{|c|c|c|c|c|c|c|c|c|}
\hline \multirow{2}{*}{$\mathbf{v}$} & \multicolumn{2}{|l|}{$\mathrm{E}_{\mathrm{v}}\left(\mathrm{cm}^{-1}\right)$} & \multicolumn{2}{|l|}{$\mathrm{R}_{\min }(\AA)$} & \multicolumn{2}{|l|}{$\mathrm{R}_{\max }(\AA)$} & \multirow{2}{*}{$\mathrm{B}_{\mathrm{v}} \times 10^{2}\left(\mathrm{~cm}^{-1}\right)$} & \multirow{2}{*}{$\mathrm{D}_{\mathrm{v}} \times 10^{8}\left(\mathrm{~cm}^{-1}\right)$} \\
\hline & & {$\left[\frac{\Delta X}{X}\right]$} & & {$\left[\frac{\Delta X}{X}\right]$} & & {$\left[\frac{\Delta X}{X}\right]$} & & \\
\hline \multirow[t]{3}{*}{0} & 157.29 & & 2.461 & & 2.612 & & 13.9 & 12.02 \\
\hline & $183.43^{\mathrm{a}}$ & $14.2 \%$ & $2.371^{\mathrm{b}}$ & $3.7 \%$ & $2.511^{\mathrm{b}}$ & $4.0 \%$ & & \\
\hline & & & $2.338^{\mathrm{c}}$ & $5.2 \%$ & $2.503^{\mathrm{c}}$ & $4.3 \%$ & & \\
\hline \multirow[t]{3}{*}{1} & 453.10 & & 2.416 & & 2.683 & & 13.724 & 10.053 \\
\hline & $548.34^{\mathrm{a}}$ & $17.3 \%$ & $2.325^{\mathrm{b}}$ & $3.9 \%$ & $2.569^{\mathrm{b}}$ & $4.4 \%$ & & \\
\hline & & & $2.311^{\mathrm{c}}$ & $4.5 \%$ & $2.553^{\mathrm{c}}$ & $5.0 \%$ & & \\
\hline \multirow[t]{3}{*}{2} & 759.88 & & 2.386 & & 2.723 & & 13.769 & 7.779 \\
\hline & $910.63^{\mathrm{a}}$ & $16.5 \%$ & $2.296^{\mathrm{b}}$ & $3.9 \%$ & $2.612^{\mathrm{b}}$ & $4.2 \%$ & & \\
\hline & & & $2.290^{\mathrm{c}}$ & $4.1 \%$ & $2.589^{\mathrm{c}}$ & $5.1 \%$ & & \\
\hline \multirow[t]{3}{*}{3} & 1082.81 & & 2.360 & & 2.760 & & 13.685 & 11.878 \\
\hline & $1270.30^{\mathrm{a}}$ & $14.8 \%$ & $2.272^{\mathrm{b}}$ & $3.8 \%$ & $2.647^{\mathrm{b}}$ & $4.2 \%$ & & \\
\hline & & & $2.272^{\mathrm{c}}$ & $3.8 \%$ & $2.620^{\mathrm{c}}$ & $5.3 \%$ & & \\
\hline \multirow[t]{3}{*}{4} & 1395.55 & & 2.340 & & 2.792 & & 13.605 & 8.554 \\
\hline & $1627.35^{\mathrm{a}}$ & $14.2 \%$ & $2.253^{\mathrm{b}}$ & $3.8 \%$ & $2.680^{\mathrm{b}}$ & $4.1 \%$ & & \\
\hline & & & $2.256^{\mathrm{c}}$ & $3.7 \%$ & $2.647^{\mathrm{c}}$ & $5.4 \%$ & & \\
\hline \multirow[t]{3}{*}{5} & 1712.20 & & 2.322 & & 2.823 & & 13.555 & 10.786 \\
\hline & $1981.78^{\mathrm{a}}$ & $13.5 \%$ & $2.236^{\mathrm{b}}$ & $3.8 \%$ & $2.709^{b}$ & $4.2 \%$ & & \\
\hline & & & $2.243^{c}$ & $3.5 \%$ & $2.672^{\mathrm{c}}$ & $5.6 \%$ & & \\
\hline
\end{tabular}

${ }^{\mathrm{a}}$ Rao, T.V.R. (1983), ${ }^{\mathrm{b}}$ Rao, T.V.R. (1983), ${ }^{\mathrm{b}}$ Lakshman and Rao's method. ${ }^{\mathrm{c}}$ Rao, T.V.R. (1983), Morse method. 
Table 4. Values of the eigenvalue $E_{v}$, the rotational constant $B_{v}$, the centrifugal distortion constants $D_{v}\left(\right.$ in $\mathrm{cm}^{-1}$ ) and the abscissas of the turning points (in $\AA$ ) of the $(2)^{2} \prod$ state of $\mathrm{CaCl}$ molecule up to $\mathrm{v}=10$

\begin{tabular}{|c|c|c|c|c|c|c|c|c|}
\hline \multirow{2}{*}{$\mathbf{v}$} & \multicolumn{2}{|l|}{$\mathrm{E}_{\mathrm{y}}\left(\mathrm{cm}^{-1}\right)$} & \multicolumn{2}{|l|}{$\mathrm{R}_{\min }(\AA)$} & \multicolumn{2}{|l|}{$\mathrm{R}_{\max }(\AA)$} & \multirow{2}{*}{$\mathrm{B}_{\mathrm{v}} \times 10^{2}\left(\mathrm{~cm}^{-1}\right)$} & \multirow{2}{*}{$\mathrm{D}_{\mathrm{v}} \times 10^{8}\left(\mathrm{~cm}^{-1}\right)$} \\
\hline & & {$\left[\frac{\Delta X}{X}\right]$} & & {$\left[\frac{\Delta X}{X}\right]$} & & {$\left[\frac{\Delta X}{X}\right]$} & & \\
\hline \multirow[t]{3}{*}{0} & 141.78 & & 2.498 & & 2.762 & & 12.989 & 15.916 \\
\hline & $167.65^{\mathrm{a}}$ & $15.4 \%$ & $2.449^{\mathrm{b}}$ & $2.0 \%$ & $2.589^{\mathrm{b}}$ & $6.6 \%$ & & \\
\hline & & & $2.445^{\mathrm{c}}$ & $2.1 \%$ & $2.592^{\mathrm{c}}$ & $6.5 \%$ & & \\
\hline \multirow[t]{3}{*}{1} & 405.64 & & 2.479 & & 2.822 & & 12.578 & 7.534 \\
\hline & $500.85^{\mathrm{a}}$ & $17.0 \%$ & $2.395^{\mathrm{b}}$ & $3.5 \%$ & $2.650^{\mathrm{b}}$ & $6.4 \%$ & & \\
\hline & & & $2.398^{\mathrm{c}}$ & $3.3 \%$ & $2.653^{\mathrm{c}}$ & $6.3 \%$ & & \\
\hline \multirow[t]{3}{*}{2} & 695.61 & & 2.447 & & 2.871 & & 12.657 & 15.707 \\
\hline & $831.25^{\mathrm{a}}$ & $16.3 \%$ & $2.364^{\mathrm{b}}$ & $3.5 \%$ & $2.695^{\mathrm{b}}$ & $6.5 \%$ & & \\
\hline & & & $2.367^{\mathrm{c}}$ & $3.3 \%$ & $2.698^{\mathrm{c}}$ & $6.4 \%$ & & \\
\hline \multirow[t]{3}{*}{3} & 987.92 & & 2.428 & & 2.910 & & 12.463 & 5.0478 \\
\hline & $1158.85^{\mathrm{a}}$ & $14.7 \%$ & $2.339^{\mathrm{b}}$ & $3.8 \%$ & $2.732^{\mathrm{b}}$ & $6.5 \%$ & & \\
\hline & & & $2.343^{\mathrm{c}}$ & $3.6 \%$ & $2.736^{\mathrm{c}}$ & $6.3 \%$ & & \\
\hline \multirow[t]{3}{*}{4} & 1277.18 & & 2.410 & & 2.948 & & 12.407 & 16.609 \\
\hline & $1483.65^{\mathrm{a}}$ & $13.8 \%$ & $2.319^{\mathrm{b}}$ & $3.9 \%$ & $2.766^{\mathrm{b}}$ & $6.5 \%$ & & \\
\hline & & & $2.323^{\mathrm{c}}$ & $3.7 \%$ & $2.770^{\mathrm{c}}$ & $6.4 \%$ & & \\
\hline \multirow[t]{3}{*}{5} & 1569.69 & & 2.395 & & 2.982 & & 12.36 & 6.3125 \\
\hline & $1805.65^{\mathrm{a}}$ & $13.0 \%$ & $2.301^{\mathrm{b}}$ & $4.0 \%$ & $2.797^{\mathrm{b}}$ & $6.6 \%$ & & \\
\hline & & & $2.305^{\mathrm{c}}$ & $3.9 \%$ & $2.801^{\mathrm{c}}$ & $6.4 \%$ & & \\
\hline \multirow[t]{3}{*}{6} & 1895.10 & & 2.380 & & 3.014 & & 12.203 & 12.43 \\
\hline & $2124.85^{\mathrm{a}}$ & $11.2 \%$ & $2.285^{\mathrm{b}}$ & $4.1 \%$ & $2.827^{\mathrm{b}}$ & $6.6 \%$ & & \\
\hline & & & $2.290^{\mathrm{c}}$ & $3.9 \%$ & $2.831^{\mathrm{c}}$ & $6.4 \%$ & & \\
\hline 7 & \multicolumn{2}{|c|}{2189.862} & \multicolumn{2}{|c|}{2.367} & \multicolumn{2}{|c|}{3.045} & 12.198 & 13.208 \\
\hline 8 & \multicolumn{2}{|c|}{2445.9} & \multicolumn{2}{|c|}{2.355} & \multicolumn{2}{|c|}{3.076} & 12.045 & 8.8098 \\
\hline 9 & \multicolumn{2}{|c|}{2800.543} & \multicolumn{2}{|c|}{2.344} & \multicolumn{2}{|c|}{3.113} & 11.926 & 16.932 \\
\hline 10 & \multicolumn{2}{|c|}{3145.834} & \multicolumn{2}{|c|}{2.334} & \multicolumn{2}{|c|}{3.138} & 11.87 & 8.7785 \\
\hline
\end{tabular}

${ }^{\mathrm{a}}$ Rao (1983), ${ }^{\mathrm{b}}$ Rao (1983), Lakshman \& Rao's method, ${ }^{\mathrm{c}}$ Rao (1983), Morse method.

The dipole moment is a fundamental electrostatic property for the description of numerous physical phenomena. Our calculated values of the static dipole moments for the considered lowest-lying electronic as a function of the internuclear distance are given in Figures 4-6. By comparing the positions of points of interaction of these curves with those of the potential energy curves (Figures 1-3) one can notice the agreement between the 3 positions of the avoided crossing of the PECs and the crossings of dipole moment curves (Table 1). This agreement may confirm the validity and the accuracy of the calculation of the studied electronic states.

\section{Conclusions}

In summary, the potential energy curves for the ground and 14 low-lying excited electronic states of $\mathrm{CaCl}$ molecule have been reported at the MRCI level. The present results show that the PECs of excited states intercross one another in several places, which make the shapes of some PECs changed. Our calculated spectroscopic parameters and vibrational levels are in good agreement with the experimental data available in literature. The comparison of these data with the theoretical results in the literature shows a very good agreement using some techniques of calculation while the agreement deteriorates for other techniques even for the same electronic state. However, there is no comparison for some molecular properties predicted for the investigated electronic states by the present work since they are calculated here for the first time. So, we believe that the present results for both the ground state and low-lying excited electronic states based, on ab initio calculations, can encourage more investigations for the molecule $\mathrm{CaCl}$. 


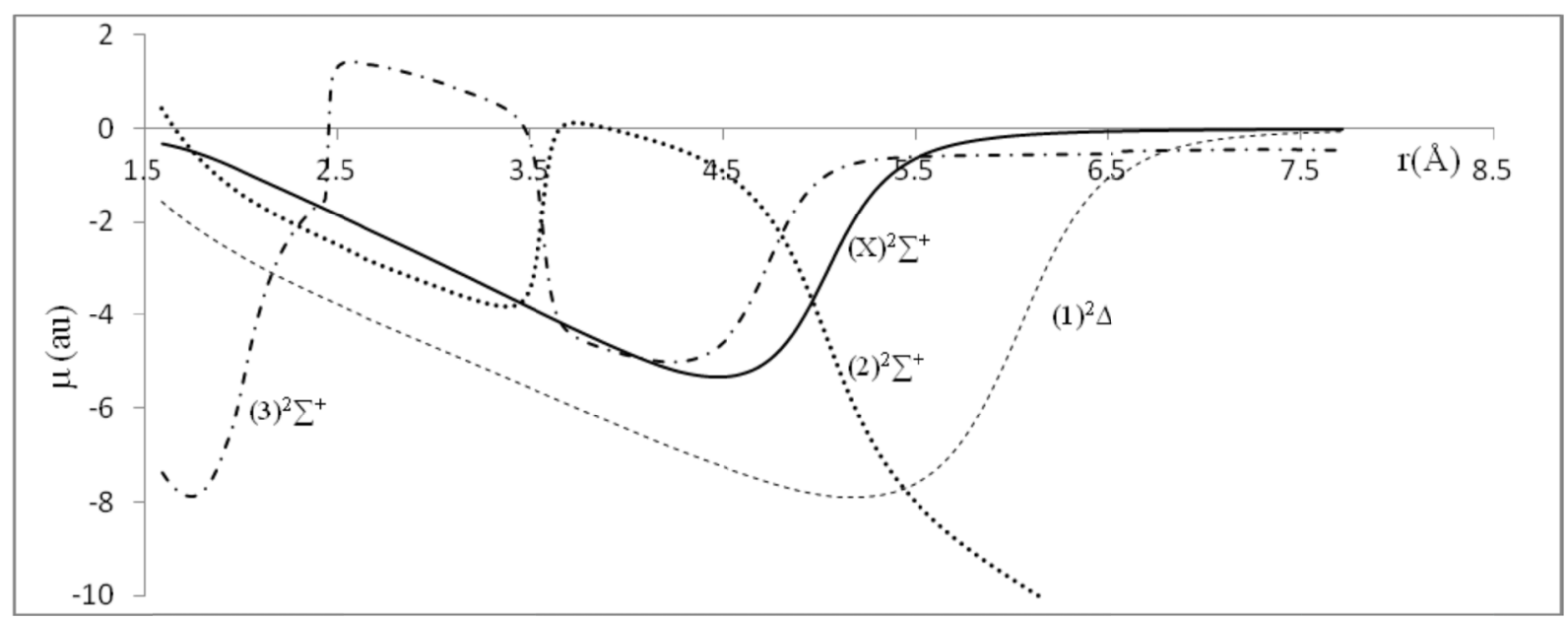

Figure 4. Permanent dipole moment, calculated by MRCI method, for the ${ }^{2} \Sigma^{ \pm}$and ${ }^{2} \Delta$ states of the molecule CaCl

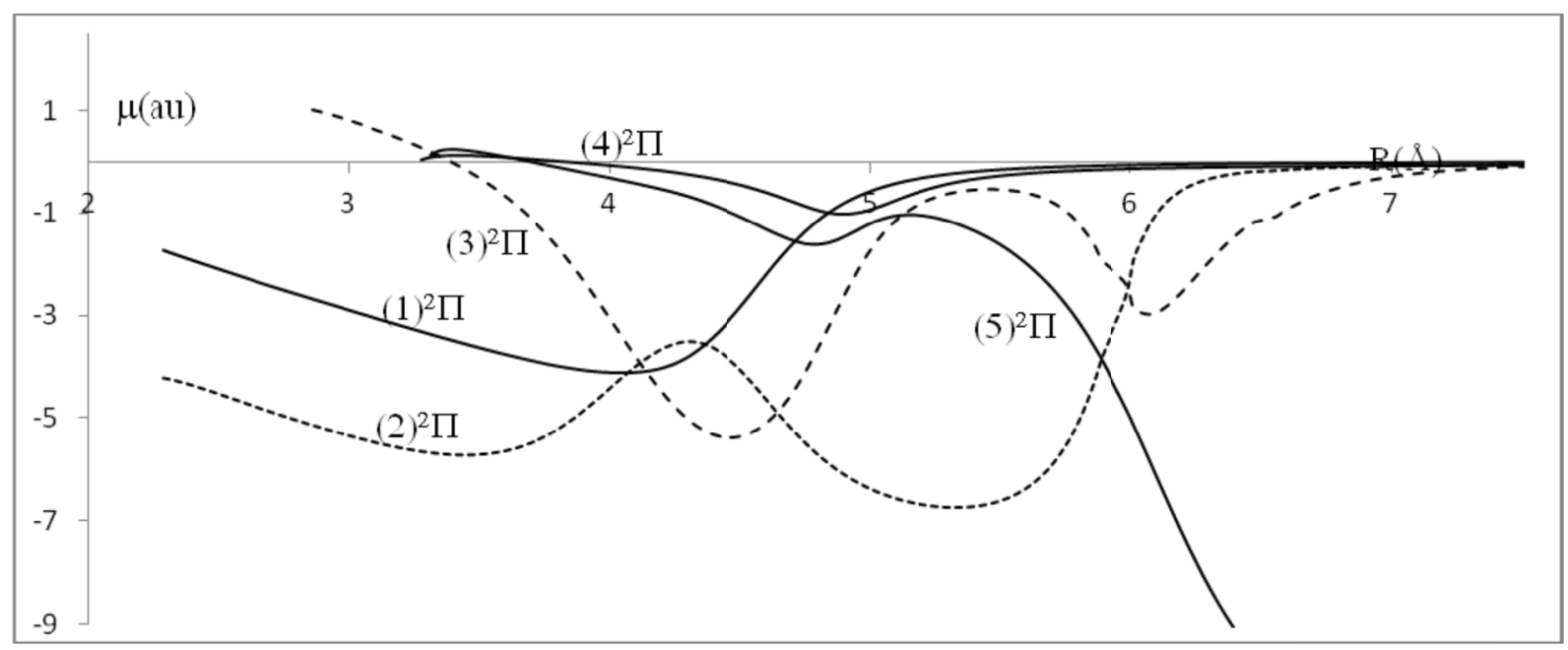

Figure 5. Permanent dipole moment, calculated by MRCI method, for the ${ }^{2} \Pi$ states of the molecule $\mathrm{CaCl}$

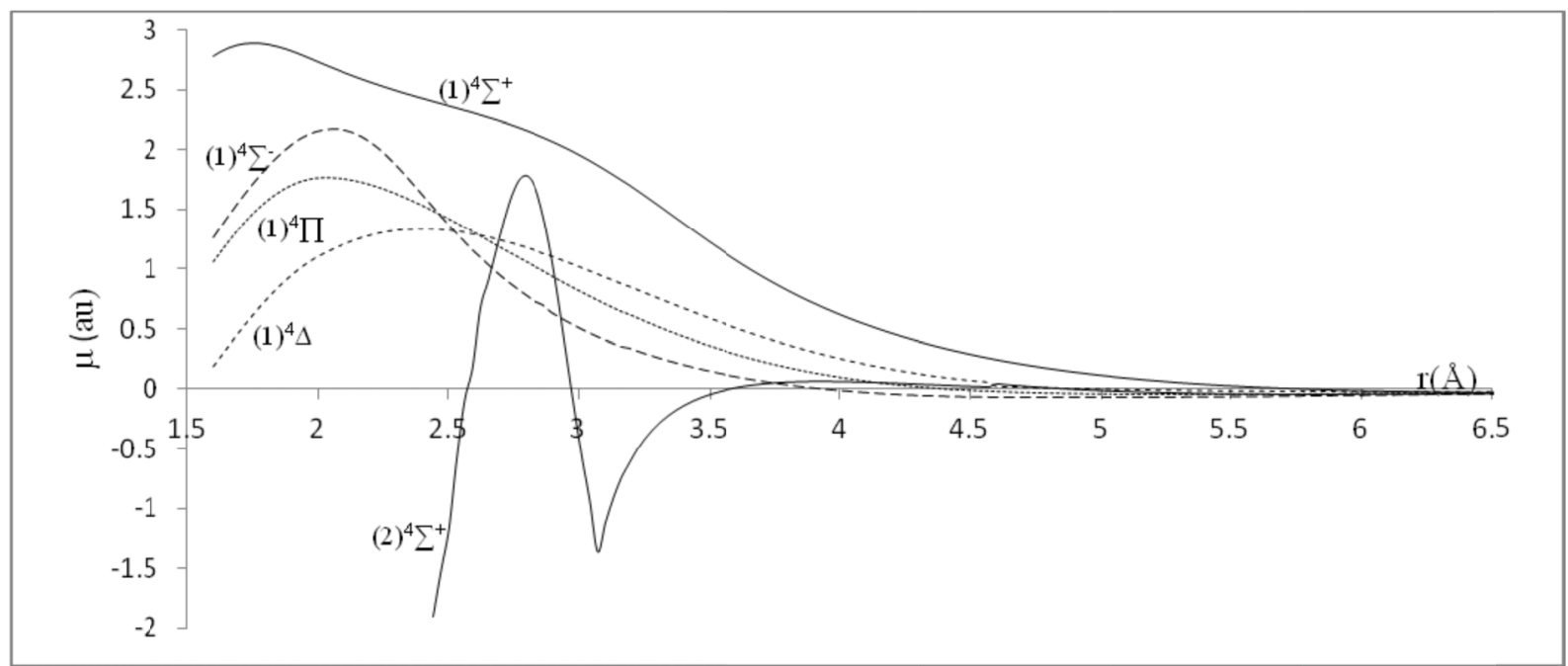

Figure 6. Permanent dipole moment, calculated by MRCI method, for the ${ }^{4} \Sigma^{ \pm},{ }^{4} \Delta$, and ${ }^{4} \Pi$ states of the molecule $\mathrm{CaCl}$ 


\section{References}

Allouche, A. R. (2011). Gabedit-A graphical user interface for computational chemistry softwares. J. Comput. Chem., 32, 174. http://dx.doi.org/10.1002/jcc.21600

Allouche, A. R., Wannous, G., \& Aubert-Fréon, M. (1993). A ligand-field approach for the low-lying states of Ca, Sr and Ba monohalides. Chemical Physics, 170, 11. http://dx.doi.org/10.1016/0301-0104(93)80087-P

Amiot, C., \& Verges, J. (1991). Laser-induced fluorescence of $\mathrm{BaCl}$ : the $\mathrm{A}^{, 2} \Delta$ state. Chem. Phys. Letters, 185 , 310. http://dx.doi.org/10.1016/S0009-2614(91)85065-1

Berg, J. M., Murphy, J. E., Harris, N. A., \& Field, R. W. (1993). Observation and Analysis of Core-Penetrating Rydberg States of Calcium Monofluoride. Phys. Rev. A, 48, 3012. http://dx.doi.org/10.1103/PhysRevA. 48.3012

Berg, L. E., Klynning, L., Martin, H., Pereira, A., \& Royen, P. (1978). Rotational analysis of the $E^{2} \Sigma-X^{2} \Sigma$ transition of CaCl. Chem. Phys. Letters, 54, 357. http://dx.doi.org/10.1016/0009-2614(78)80117-1

Bündgen, P., Engels, B., \& Peyerimhoff, S. D. (1991). An MRD-CI study of low-lying electronic states in CaF. Chem. Phys. Lett., 176, 407. http://dx.doi.org/10.1016/0009-2614(91)90228-2

Childs, W. J., Goodman, L. S., Nielsen, U., \& Pfeufer, V. (1984). Electric-dipole moment of $\mathrm{CaF}\left(\mathrm{X}^{2} \Sigma^{+}\right)$by a molecular beam, laser-rf, double resonance study of stark splitting. J. Chem. Phys., 80, 2283. http://dx.doi.org/10.1002/1.447005

D'incan, J., Effantin, C., Bernard, A., Verges, J., \& Barrow, R. F. (1991). Laser spectroscopy of the CaOH A²П$\mathrm{X}^{2} \Sigma^{+}$. Phys. B, 24, 127.

Domaille, P. J., Steimle, T. C., Wong, N. B., \& Harris, D. O. (1977). High-Resolution laser excitation spectroscopy analysis of the $\mathrm{E}^{2} \Sigma^{+}-\mathrm{X}^{2} \Sigma^{+}$system of CaCl. J. mol. Spectry, 65, 354. http://dx.doi.org/10.1016/ 0022-2852(77)90275-2

Dykstra, C. E. (1977). An examination of Brueckner condition for selection of molecular-orbitals in correlated wavefunctions. Chem. Phys. Lett., 45, 466. http://dx.doi.org/10.1016/0009-2614(77)80065-1

Ernst, W. E., Kindt, S., Nair, K. P. P., \& Torring, T. (1984). Determination of the ground state dipole moment of $\mathrm{CaCl}$ from molecular-beam laser microwave double resonance measurements. Phys. Rev., A29, 1158. http://dx.doi.org/10.1103/PhysRevA.29.1158

Handy, N. C., Pople, J. A., Head-Gordon, M., Raghavachari, K., \& Trucks, G. W. (1989). Size-consistent Brueckner Theory Limited to Double Substitutions. Chem. Phys. Lett., 164, 185. http://dx.doi.org/10.1016/ 0009-2614(89)85013-4

Harris, N. A., \& Jungen, Ch. (1993). Rydberg states of calcium fluoride. Phys. Rev. Lett., 70, 2549-2552. http://dx.doi.org/10.1103/PhysRevLett.70.2549

Huber, K. P., \& Herzberg, G. (1978). Molecular spectra and molecular structure (Vol.4, Van Nostrand, Princeton, 1978).

Kaledin, L. A., Bloch, J. C., Mccarthy, M. C., \& Field, R. W. (1999). Analysis and Deperturbation of the $A^{2} \Pi$ and $\mathrm{X}^{2} \Sigma^{+}$States of CaF. J. Mol. Spectrosc., 197, 289. http://dx.doi.org/10.1006/jursp.1999.7909

Klynning, L., \& Martin, H. (1981). On the interaction between the $\mathrm{A}^{2} \Pi$ and $\mathrm{B}^{2} \Sigma^{+}$states of $\mathrm{CaCl}$ molecule. Phys. Scr., 24, 25. http://dx.doi.org/10.1088/0031-8949/24/1A/006

Knowles, P. J., \& Werner, H. J. (1985). An efficient second-order MC SCF method for long configuration expansions. Chem. Phys. Lett., 115, 259. http://dx.doi.org/10.1016/0009-2614(85)80025-7

Kobeissi, H., Korek, M., \& Dagher, M. (1989). On the computation of diatomic centrifugal distortion constants: Exact solutions for initial value problems. J. Mol. Spectrosc., 138, 1. http://dx.doi.org/10.1016/0022-2852 (89)90092-1

Korek, M. (1999). A one directional shooting method for the computation of diatomic centrifugal distortion constants. Comput. Phys. Commun., 119, 169. http://dx.doi.org/10.1016/S0010-4655(98)00180-5

Korek, M., \& Kobeissi, H. (1992). Highly accurate diatomic centrifugal distortion constants for high orders and high levels. J. Comput. Chem., 13, 1103. http://dx.doi.org/10.1002/jcc.540130909 
Langhoff, S. R., Bauschlicher, C. W., \& Partridge, H. (1986). Theoretical dissociation energies for the alkali and alkaline-earth monofluorides and monochlorides. J. Chem. Phys., 84, 1687. http://dx.doi.org/10.1063/1.450 466

Menzinger, M. (1974). Dynamics of the electronically chemiluminescent $\mathrm{Ca}+\mathrm{X}_{2}\left(\mathrm{~F}_{2}, \mathrm{CL}_{2}, \mathrm{Br}_{2}\right)$ reactions. Chem. Phys., 5, 350. http://dx.doi.org/10.1016/0009-2614(74)90267-X

Molpro. (ND). Molpro (a package of ab-intio programs written by Werner et al.).

Murphy, J. E., Berg, J. M., Merer, A. J., Harris, N., \& Field, R. W. (1990). Rydberg States and ionization Potential of Calcium Monofluoride. Phys. Rev. Lett., 65, 1861. http://dx.doi.org/10.1103/PhysRevLett.65.1861

Rao, T. V. R., Reddy, R. R., \& Reddy, A. S. (1983). Potential energy curves and bond dissociation energies of $\mathrm{CaF}, \mathrm{CaCl}, \mathrm{CaI}$ and CaH. J. Mol. Struct. (Theochem.), 105, 249. http://dx.doi.org/10.1016/0166-1280(83) 80203-6

Rice, S.F., Martin, H., \& Field, W. (1985). The Electronic Structure of the Calcium Monohalides. A Ligand Field Approach. J. Chem. Phys., 82, 5023-5034. http://dx.doi.org/10.1063/1.448676

Torring, T., Ernst, W. E. J., \& Kandler, J. (1989). Energies and electric dipole moments of the low lying electronic states of the alkaline earth monohalides from an electrostatic polarization model. J. Chem. Phys., 90, 4927-4932. http://dx.doi.org/10.1063/1.456589

Verges, J., Effantin, C., Bernard, A., Topouzkhanian, A., Allouche, A. R., incan, J. D., \& Barrow, R. F. (1993). The (B) ${ }^{2} \Delta$ state of CaF . J. Phys. B, 26, 279. http://dx.doi.org/10.1088/0953-4075/26/2/011

Werner, H. J., \& Knowles, P. J. (1985). A second order multiconfiguration SCF procedure with optimum convergence. Chem. Phys., 82, 5053. http://dx.doi.org/10.1063/1.448627

Yang, C. L., Zhang, X., \& Han, K. L. (2004). Theoretical study on analytical potential function and spectroscopic parameters for CaF molecule. J. Mol. Struct. (Theochem.), 678, 183. http://dx.doi.org/10.1016/j.theochem. 2004.03.021

\section{Copyrights}

Copyright for this article is retained by the author(s), with first publication rights granted to the journal.

This is an open-access article distributed under the terms and conditions of the Creative Commons Attribution license (http://creativecommons.org/licenses/by/3.0/). 